\title{
Biopolítica y democracia como estilo de vida: una mirada desde la psicología social
}

\author{
Biopolitics and Democracy as a Lifestyle: \\ A View from Social Psychology
}

Álvaro Díaz-Gómez ${ }^{1} \bowtie$, Olga Lucía Carmona², Liliana Andrea Salamanca ${ }^{3}$

\author{
${ }^{1}$ Universidad Tecnológica de Pereira, Colombia \\ 2 Universidad Tecnológica de Pereira, Colombia \\ ${ }^{3}$ Universidad Tecnológica de Pereira, Colombia
}

$\triangle \mathrm{Cl} .27$ n. ${ }^{\circ}$ 10-02, Pereira, Risaralda, Colombia. Correo electrónico: adiaz@utp.edu.co

Recibido: 8 de marzo del $2017 \quad$ Aprobado: 16 de febrero del 2018 Disponible en línea: 1 de abril de 2018

Cómo citar este artículo: Díaz-Gómez, A., Carmona, O. L. y Salamanca, L. A. (2018). Biopolítica y democracia como estilo de vida: una mirada desde la psicología social. Pensando Psicología, 14(23). doi: https://doi.org/10.16925/pe.v14i23.2263

\section{Resumen}

Propósito: reflexionar sobre dos maneras de asumir la democracia (como forma de gobierno y como estilo de vida), con el fin de pensar desde la psicología social —asumida como una psicología que es política — su relación con la biopolítica, desde un caso particular que se presentó en Colombia, conocido como "los falsos positivos".

Tema: la posibilidad de pensar desde la psicología social la vida que se controla políticamente, por lo cual se asume desde el discurso de la biopolítica.

Desarrollo: se realiza una contextualización con el propósito de reconocer que la psicología social es política dado el uso del conocimiento que produce, por lo que no le pueden ser ajenos problemas que emergen en la construcción de la democracia, y es así plausible el abordaje de su expresión como estilo de vida en relación con lo que es como forma de gobierno. Desde esta se instauran dispositivos biopolíticos para el control de la vida, y son ejemplo de lo anterior las ejecuciones extrajudiciales que se implementaron en Colombia, conocidas como "falsos positivos".

Conclusiones: se visibiliza aún cómo la psicología, y en particular la psicología social, es política dados los usos que se le dan al conocimiento. Por tanto, si este se piensa en términos de las mayorías populares, puede aportar en la construcción de una sociedad democrática, en la que esta no sea solo un proceso estructural de gobierno, sino que se despliegue como un ethos cultural en la vida cotidiana. Tal vez así se generen procesos de resistencia a la biopolítica y emerja una psicopolítica.

Palabras clave: biopolítica, democracia como estilo de vida, democracia como forma de gobierno, falsos positivos, psicología social, política. 


\title{
Biopolitics and Democracy as a Lifestyle: A View from Social Psychology
}

\begin{abstract}
Purpose: To reflect on two ways of adopting democracy, as a form of government and as a way of life, in order to establish its relationship with biopolitics from social psychology —as a psychology that is political — based on a particular case that occurred in Colombia known as "false positives."

Theme: The possibility of thinking about a life that is politically controlled from the perspective of social psychology, for which it is assumed from the discourse of biopolitics.

Elaboration: A contextualization is provided for recognizing that social psychology is political given the use of the knowledge it produces, so that problems that emerge in the construction of democracy cannot be alien to it and, thus, it is plausible to approach its expression as a lifestyle in relation to what it is as a form of government. From this, biopolitical devices for life control are established, and the extrajudicial executions implemented in Colombia, known as "false positives," are an example of this.

Conclusions: It is still visible how psychology, particularly social psychology, is political because of the way knowledge is used. Therefore, if thought in terms of popular majorities, knowledge may contribute to the construction of a democratic society, in which it is not only a structural governmental process but also deployed as a cultural ethos in daily life. Perhaps this is how processes of resistance to biopolitics are generated and a psychopolitics emerges.
\end{abstract}

Keywords: biopolitics, democracy as a way of life, democracy as a form of government, false positives, social psychology, politics

\section{Biopolítica e democracia como estilo de vida: um olhar a partir da psicologia social}

\section{Resumo}

Propósito: refletir sobre duas maneiras de assumir a democracia: como forma de governo e como estilo de vida, com o objetivo de pensar a partir da psicologia social — entendida como uma psicologia que é política — sua relação com a biopolítica, a partir de um caso particular que ocorreu na Colômbia e ficou conhecido como "os falsos positivos".

Tema: a possibilidade de pensar, a partir da psicologia social, a vida que é politicamente controlada e por isso é assumida a partir do discurso da biopolítica.

Desenvolvimento: realiza-se uma contextualização com o objetivo de reconhecer que a psicologia social é política, dado o uso do con hecimento que produz, porque os problemas que emergem na construção da democracia não podem ser alheios a ela; assim, é plausivel a abordagem de sua expressão como estilo de vida em relação com o que é como forma de governo. A partir dela dispositivos biopolíticos para o controle da vida são instaurados e as execuções extrajudiciais que foram implementadas na Colômbia, conhecidas como "falsos positivos", são exemplos disso.

Conclusões: visibiliza-se, ainda, como a psicologia e, em particular, a psicologia social, é política considerando os usos que dão ao conhecimento. Portanto, se pensamos o conhecimento em termos das maiorias populares, ele pode contribuir na construção de uma sociedade democrática na qual esta não seja apenas um processo estrutural de governo, mas que se desdobre como um ethos cultural na vida cotidiana. Talvez assim sejam gerados processos de resistência à biopolítica e seja emergida uma psicopolítica.

Palavras-chave: biopolítica, democracia como estilo de vida, democracia como forma de governo, falsos positivos, psicologia social, política. 


\section{Introducción}

¿Desde qué psicología social hablamos? ¿Es la psicología social política? En el presente texto asumiremos que sí, sin que ello implique la negación de la psicología política.

Hasta mediados de la década de los setenta del siglo pasado fue hegemónica la idea de la neutralidad de las ciencias. Esto empezó a cambiar en la psicología, y en particular en la psicología social, cuando se planteó la crítica sobre el papel que del conocimiento hace el psicólogo en la sociedad y con esto su rol transformador de esta - no solo como profesional, sino como ciudadano interesado por el bien común (por la política)-, del carácter histórico del conocimiento, su procesualidad y, por tanto, de la crítica al concepto universal de "objetividad". Las reflexiones que se realizaron sobre estos tópicos se expresaron en lo que se conoció como "la crisis de relevancia de la psicología social” (Baró, 1988; Montero, 2004).

Como consecuencia de esta crisis de relevancia se empezó a reconocer que todo conocimiento es político y se hace uso político de él. Por tanto, no existe neutralidad del conocimiento en sí, ni del investigador que lo produce o el profesional que lo reproduce.

Se deriva de lo anterior que la psicología, y con ello la psicología social, son políticas en razón del uso social del conocimiento que producen, los temas que privilegian investigar y las maneras como lo realizan, así como la focalización de los grupos a los cuales van dirigidas.

De tal manera, las ideas que aquí desarrollamos se ubican en una psicología social, que es política por su perspectiva de compromiso social y su focalización en un grupo poblacional determinado (jóvenes asesinados en condición de indefensión), pero que reconoce la especificidad de la psicología política - dado su objeto de estudio en pluralidad - sin confundirse con ella.

Desde este punto de vista, se abordan las categorías "democracia" - ya asumida por algunos estudiosos en relación con la psicología social (Brandao, 2010; Bruno y Barreiro, 2015; Porras, 2010)-, y "biopolítica" - asumida también desde la psicología social (Andrade-Salazar 2013; Cruz, 2017; Grajales, 2014; Pastor, 2009).

\section{La democracia como estilo de vida, escenario para pensar la educación para la democracia}

Pensar la democracia como estilo de vida no es nuevo. De hecho, Dewey (1995) lo planteó así: "Una democracia es más que una forma de gobierno, es primariamente un modo de vivir asociado, de experiencia comunicada conjuntamente" (p. 82). Esta acepción de la democracia ha sido poco abordada, por lo que sus producciones teóricas no forman parte de los discursos hegemónicos. Aun así, su potencia creadora y complementaria a la democracia como forma de gobierno se reconoce y profundiza de manera progresiva.

En tal sentido, se encuentran argumentos sugerentes como los de Mora (2012):

La democracia es una forma de vida y convivencia entre sujetos que conforman un Estado o Nación. Por ello, la democracia es una construcción, una hechura humana que puede ser desarrollada en el tiempo con altos niveles de participación o sencillamente puede ser limitada o destruida mediante las tradicionales formas de representación.

Esto implica libertad para discernir, ser y participar, mientras de forma simultánea se pone de manifiesto el ejercicio de la ciudadanía y, con ello, la constitución de un sujeto político.

Ahora bien, es pertinente reconocer lo que sobre estilos de vida nos propone Hederich (2007). En sus indagaciones encuentra que, desde la psicología, la primera aproximación a la noción de "estilo" se encuentra en un trabajo de Lewin, en 1935, quien la asumió como una expresión de la personalidad y la disposición al uso de ciertas habilidades cognitivas. En esta misma línea, Allport (1937) se refiere a "estilos de vida" que identifican cierto tipo de comportamientos. Por tanto, más que banal, la noción de estilo de vida se torna fundamental para pensar la democracia no solo referida a la dimensión macropolítica y estructural del Estado, sino a fin de indagarla en lo microsocial, en lo procesual de la vida diaria.

Dado lo anterior, recogemos la propuesta de entender el estilo "como un conjunto de regularidades consistentes en la forma de la actividad humana que se lleva a cabo por encima del contenido, esto es, 
de los dominios propios de la actividad" (Hederich, 2007, p. 23). Este mismo autor plantea cuatro características de los estilos: su carácter diferenciador (lo que permite establecer características distintivas); su relativa estabilidad en las personas; la posibilidad integradora de diferentes dimensiones del sujeto; y su carácter neutral en cuanto no se puede valorar un estilo por encima de otro.

Desde estos planteamientos podemos entender la democracia como estilo de vida en cuanto a una manera complementaria de abordar en el plano teórico y existencial la democracia como forma de gobierno. Este abordaje se concreta en actividades humanas cotidianas que se expresan de forma individual y adquieren un carácter distintivo con cierta estabilidad provisional que integra dimensiones de la política como, por ejemplo, la ética y la moral, pues "sólo es posible producir individuos democráticos mediante la multiplicación de las instituciones, los discursos, las formas de vida que fomentan la identificación con los valores democráticos" (Mouffe, 2000, p. 109).

Díaz y Valencia (1996a; 1996b) plantean que la democracia se puede abordar desde dos expresiones: la primera, como forma de gobierno referida a la política y al ámbito de la institucionalidad, en la que se delega en los políticos su concreción; la segunda, como estilo de vida que hace posible,

Comprender la democracia, como algo que a manera de tejido social, vamos construyendo desde nuestro nivel de injerencia individual en el espacio de nuestra vida cotidiana, lo que implica hacernos protagonistas de las deliberaciones sociales y partícipes de los procesos organizativos que pueden permitir que vayamos aumentando los niveles de incidencia y poder desde propuestas negociadas para influir en actos de gobierno. (1996b, p. 55)

Estas acepciones son niveles y formas a través de las cuales se ejerce democracia, por lo que es posible entenderlas de manera diferenciada y diferenciable, así como caracterizarlas por separado. No tanto porque sean excluyentes, sino porque dadas las maneras como se concretan, asumen particularidades: "La democracia liberal (...) entendida como régimen concierne al ordenamiento simbólico de las relaciones sociales y es mucho más que una mera 'forma de gobierno'. Es una forma específica de organizar políticamente la coexistencia humanas" (Mouffe, 2000, p. 36).
Como parte de la caracterización de la democracia en cuanto estilo de vida se plantean:

Cuatro ideas centrales que a manera de vectores sociales, marcan unas direcciones susceptibles de tener en cuenta, a la hora de asumir nuevas formas de pensar la democracia: la cotidianidad como espacio-tiempo en el cual se vive la democracia como estilo de vida; la socialización en cuanto hipertexto que media la constitución de estilos de vida democráticos; lo público y lo privado, como dos escenarios que se deben vivir en democracia; la educación para la democracia, como eje intencional en la constitución de sujeto democrático. (Díaz, 1999, p. 48)

Estos vectores permiten reconocer más cercanía de los sujetos/ciudadanos con la democracia, y explicitan cómo es una construcción cotidiana y no solo un acto formal en las urnas, lo que implica la participación de diferentes agencias y agentes instituyentes de ethos democráticos, por lo que la vida democrática no se debe expresar solo en los espacios públicos sino también en los privados, en los que la relación interpersonal media y constituye las relaciones sociales. No obstante, en cuanto estas no son dadas genéticamente se requiere su construcción colectiva, participativa e histórica, vía la educación en una de sus acepciones: la educación para la democracia (Díaz, 2000a; Díaz, 2000b, Díaz, 2003; Seoane, Mougán y Lago, 2009).

Educar para la democracia implica contar con espacios y oportunidades que promuevan y permitan aprender habilidades y concepciones democráticas y ciudadanas; asumir los deberes y derechos que socialmente se han acumulado como legado cultural; participar activamente en los procesos públicos; y constituirse como nuevo sujeto en los aspectos simbólicos, éticos y morales que le confieran un sentido de identidad y pertenencia a una comunidad.

Sin embargo, introducir el término "constituirse como nuevo sujeto", conduce a que se plantee cómo se da esto, reconociendo el papel fundamental de la socialización y por esta vía de la educación, para preguntar: ¿cómo se aprende a ser ciudadano? ¿Qué ocurre con el sistema de relaciones sociales e institucionales en este proceso? O bien, ¿cuál es su visión de la educación con miras al ciudadano del futuro?

Así, entonces, es necesario reconocer de manera cada vez más apremiante la articulación de los procesos educativos en sus diferentes modalidades (formal, 
no formal e informal) con la construcción de ciudadanía, lo que impone a estos procesos formativos algunos desafíos propuestos por Castillo y Osorio (1997): permitir que circulen, para todos, los conocimientos y la apropiación de los lenguajes que vehiculizan la información social necesaria para la participación ciudadana; formar en valores que potencien capacidades y competencias para realizarlos responsable y críticamente en los diferentes ámbitos de la vida social, formar ciudadanos autónomos y críticos; superar el individualismo para encarar de manera comunicativa los problemas de una comunidad; relacionar lo individual con lo universal, promoviendo la apertura del ciudadano a acciones colectivas; ejercer colectivamente el poder de control sobre el gobierno y resolver pacíficamente los conflictos; y cuestionar la idea de un ciudadano abstracto.

¿Cómo lograr lo anterior? Aquí surge una tensión con respecto a cómo enseñar para la vida, con el fin de ser humano, qué enseñar y quién debe educar al sujeto ético y moral. Si lo que se pretende es constituir subjetividad democrática, ¿puede parcelarse en un currículo, como ocurre con las matemáticas o las ciencias sociales? Dado que la autoproducción humana y la constitución de subjetividad son espacios de formación caracterizados por la pluralidad, el azar y el caos, no existe una receta, ni una ruta única mediante la cual se pueda ofrecer la realización plena de las mujeres y hombres que avanzan hacia procesos de mayor humanización. La creatividad, la exploración y la iniciativa individual y colectiva son el horizonte para proponer y desarrollar concepciones pedagógicas, educativas y didácticas que ayuden a avanzar en esta pretensión.

Por tanto, una característica de los procesos de educación de sujetos democráticos es su transversalidad, en cuanto no corresponden solo a un nivel de la educación, a una modalidad específica, a una clase de currículum, ni tampoco a una agencia educativa determinada y, en consecuencia, a unos agentes socializadores determinados, sino a todos y cada uno de ellos. La educación para la democracia, además de transversal, nos permite proponer el símil del hipertexto, en el que desde múltiples ventanas entro y salgo, me meten y me sacan, de y para discursos y prácticas diferentes, pero que me forman como una totalidad.

Si bien es cierto que no existe una única agencia ni un único agente educativo para educar en democracia, cada una de las personas e instituciones que en ella participan deben pensar y pensarse como sujetos constructores de humanidad, a fin de precisar los ámbitos específicos desde los cuales van a intervenir en este proceso de construcción social.

Sin que sean fórmulas mágicas sino referentes posibles de asumir, se puede hacer uso de: deliberación con respecto a dilemas morales, resolución de conflictos presentados en el grupo al que se pertenece, la construcción de acuerdos consensuados, la concreción de negociaciones, el desarrollo de formas compartidas de comprensión y la apropiación de conceptos éticos (Castillo y Osorio, 1997).

\section{Una opción de educación para la democracia como estilo de vida: el abordaje de lo político}

Retomaremos algunos planteamientos formulados por Mouffe $(1999,2000)$ en cuanto nos permiten comprender dimensiones para la educación en democracia. Ella nos hace ver una diferencia conceptual y vivencial de dos categorías distintas pero complementarias: "la política" y "lo político". La primera hace referencia a los mecanismos, a las formas mediante las cuales se establece un orden y se organiza la existencia humana que siempre se presenta en condiciones conflictivas; y la segunda se refiere a una cualidad de las relaciones entre las existencias humanas, y se expresa en la diversidad de las relaciones sociales:

Con ese fin propone distinguir entre "lo político", ligado a la dimensión de antagonismo y de hostilidad que existe en las relaciones humanas, antagonismo que se manifiesta como diversidad de las relaciones sociales, y "la política", que apunta a establecer un orden, a organizar la coexistencia humana en condiciones que son siempre conflictivas, pues están atravesadas por "lo político". (Mouffe, 1999, p. 14)

Teórica y pragmáticamente ha existido mayor preocupación por la mirada y la cualificación de los mecanismos y procedimientos para vivenciar la democracia, es decir, la política, lo que ha implicado colocar el énfasis en la democracia como forma de gobierno, de modo que se deja en un segundo lugar el abordaje de cualidades propias de lo político como son la contradicción y la diferencia, constitutivas de una mirada a la democracia como estilo de vida. Sobre esto debe centrarse un proceso de formación para la democracia en perspectiva ciudadana. 
De acuerdo con Mouffe (1999), lo común entre la política y lo político es la significación que desde sus raíces etimológicas tienen estas palabras: la polémica, propia de lo político y que surge de la diferencia; y la pretensión necesaria de vivir juntos, característica de la política. Pero no se trata, dice ella, de privilegiar el vivir juntos propio de la polis, dejando de lado el pólemos, condición del antagonismo y el conflicto.

Por otra parte, y de manera complementaria, se presentan el antagonismo y el agonismo. El primero tienen una relación directa con el enemigo, ya que este se asume como aquel con quien encontrándome en una posición opuesta que se ha tornado irreconciliable, se asume como alguien a quien se debe eliminar, lo que no quiere decir quitarle la vida (aunque en el caso de los falsos positivos se asumió de manera radical esta opción). Esta eliminación se puede dar por vía de la indiferencia que se asume como silencio, no comunicación. Esta incomunicación es una vía teórica derivada de la propuesta de Arendt, para quien aquel que no entra en procesos de comunicación, no existe. Cada uno de nosotros nace ante el otro, en tanto proceso de natalidad, cuando se hace visible mediante el lenguaje (Arendt, 1954; Mélich, 1994).

El agonismo, por su parte, hace referencia y se relaciona con el adversario, y este se asume como alguien, otro diferente a mí, pero a quien considero interlocutor válido, por lo que no se verá como un enemigo a abatir, sino como un otro de legítima existencia a quien se tolera, con quien se debaten ideas con vigor y no se le niega el derecho a defender las suyas.

Por tanto, se puede derivar que en procesos de educación para la democracia se debe afianzar el espíritu agonal que se erige en fundamento de esta. Cuando esto no ocurre y se propicia el antagonismo, se prevén —retomando los planteamientos de Mouffe (1999) - las siguientes consecuencias: se pierden las diferencias entre adversarios; se borra la identidad de derecha e izquierda; se pierde toda referencia a las diferencias; se crea una "república del centro"; no se presenta el adversario político (este es un competidor); se crea un terreno propicio para los movimientos extremos, bien sean de derecha o de izquierda, religiosos, étnicos o nacionalistas; al no encontrar formas de identidad colectiva, se buscan otras formas de identidad; se pierde la noción de adversario por la de enemigo al que se debe acabar; y se pone en peligro la democracia al no existir consenso sobre instituciones y valores.
En contextos como el colombiano, para los guerreros, los que asumen la vía militar como opción con el fin de participar socialmente en el ámbito de la política, lo que predomina es la imagen del otro como enemigo, a quien de múltiples formas intentan destruir o acabar aun desde las formas más radicales, como la de matar al otro por la imposibilidad de asumirlo en su diferencia constructora. En cada acto de asesinato, el asesino se asesina a sí mismo en tanto que para ser se requiere del otro; y si lo aniquila, se pierde el contradictor, el referente, la otredad.

\section{Biopolítica y democracia como forma de gobierno}

Las relaciones dualistas establecidas por el pensamiento moderno dejaron a la política habitar solo en el Estado como forma de gobierno ( $y$ no en la sociedad civil como estilo de vida), de manera que lo engrandecieron a través de su concentración de poder.

Así, las distintas construcciones de la práctica y la teoría política desembocaron en modelos de gobierno que pasaron desde aquellos que quisieron pacificar los ataques del hombre contra el hombre, hacia otros que buscaron construir acuerdos entre hombres libres e iguales ante la ley, hasta llegar a experiencias de dictaduras para igualar lo diferente.

Estos modelos, que siempre tienen en la base un modo de producción, se han transformado progresivamente; así, el capitalismo compitió - como su esencia lo indica- por concentrar el poder, y lo obtuvo a través de una de sus expresiones: el liberalismo económico. Sin embargo, la subsistencia del modelo económico y de su expresión política generaron poco a poco dispositivos de control y poder, lo que propició la emergencia de prácticas biopolíticas que posteriormente se reflexionan desde el concepto de biopolítica, y se critican los discursos naturalizados de universalidad, racionalidad, contrato social y consenso entre individuos, propios del liberalismo (Bartolomé, 2012; Toscano, 2008).

Una breve ubicación sobre qué entender por biopolítica nos lleva al plano etimológico, en el que el prefijo bio- se entiende como "vida"; y política se asume como "gobierno". Este, que puede ser un comienzo de comprensión, es insuficiente frente al desarrollo teórico que lo sustenta. Así, Esposito (2006) expone dos opciones de comprensión: bio-, leído como "forma de vida"; y bio- como zoe, "como simple mantenimiento de lo biológico", por lo que la biopolítica permite hacer un tránsito entre el bios y el 
zoe por medio de la técnica. Esto conduce a preguntar: “¿Debe entenderse que la vida gobierna la política, o que bien la política gobierna la vida? ¿Se trata de un gobierno de o sobre la vida?" (Esposito, 2006, p. 26).

Esta relación entre vida y política la aborda Foucault (1984; 2008) mediante el análisis de las formas de poder y de disciplinamiento del cuerpo (biopoder), y las formas de gobernar y controlar las poblaciones (biopolítica) que asume el soberano para el cumplimiento de sus objetivos, de modo que hace suya la máxima - en cuanto derecho- "de hacer morir o de dejar vivir". Así, condiciona cuerpos, vidas y libertades con el fin de detener su potencia. De esta manera, la biopolítica busca "gobernar la vida entera, desde sus ritmos de crecimiento (fertilidad, nupcialidad, natalidad y mortalidad) hasta la actividad de cada individuo, tanto en sus horas de trabajo como en las de ocio" (Ugarte, 2005, p. 8).

La biopolítica se puede entender como el cruce de dos polos (vida/política) que se entrelazan de manera constituyente y tienen la potencia de conservar la vida mediante la administración cuidadosa de los cuerpos y la creación de nuevas formas para la sujeción y control de las poblaciones. Esto es importante en tanto condición de posibilidad para que la vida pueda mantenerse con "vida" y se reflexione sobre la necesidad de la relación entre la vida y la política como instituyentes en el devenir del sujeto.

Esposito plantea lo siguiente: "El bíos es el objeto y el sujeto del conflicto y, por ende, de la política por él moldeada" (Esposito 2006, p. 45); por tanto, se trata de entender que en el contexto moderno se genera una especie de "estatización de lo biológico", en la que el cuerpo se asume como una realidad biopolítica sobre la que se deben ejercer nuevas formas de dominación. Así, las teorías psicológicas, la educación, la política - por ejemplo- son dispositivos de vigilancia y control sobre el cuerpo y la vida. Esta "deja de ser un resultado del azar, si se quiere el regalo concedido por la divinidad, para convertirse en una consecuencia de la intervención política y por ello es puesta en juego en la práctica cotidiana del poder" (Ugarte, 2005, p. 10).

En ese orden de ideas, la biopolítica no es una práctica inocente, pues esta "procura afirmar y aumentar el poder del Estado teniendo por objeto la población, por saber estratégico la economía política y por herramienta técnica los dispositivos de seguridad" (Toscano, 2008, p. 78). El sistema capitalista impregna su propio sentido economicista a la política, a las formas de gobierno y así a las formas de control sobre el cuerpo social y los cuerpos que lo componen, creando las condiciones para que aflore y se mantenga el miedo:

Se trata de gobernar la vida entera. Cabe decir que los ciudadanos, además de ser los depositarios de un conjunto de derechos y deberes, son también sujetos en el doble sentido del término "sujeto", es decir, independientes del poder al tiempo que necesitan todos los recursos que este proporciona para realizar su independencia. ( Ugarte, 2005, p. 12)

\section{Los falsos positivos en tiempos de seguridad democrática}

Desde la anterior ubicación general sobre qué entender por biopolítica y algunos de sus alcances, procederemos a argumentar cómo este concepto que aborda nuevas formas de dominación sobre la vida es pertinente para el análisis de la sociedad colombiana y las prácticas gubernamentales contemporáneas, con base en un caso: los denominados "falsos positivos" o crímenes de lesa humanidad. En este caso se asume lo biopolítico "como un modo de acercamiento analítico a lo social que tiene por objeto adentrarse en el proceso mismo en el que el ordenamiento de cada forma de vida tiene lugar" (Mendiola, 2009, p. 9).

Dado que Colombia vivió un conflicto interno armado de más de 50 años, han sido diferentes las políticas formuladas por los gobiernos de turno con el fin de mantener el orden, la seguridad y el control. No obstante, la política de seguridad democrática, desarrollada del 2002 al 2010 en la presidencia de Álvaro Uribe, sobrepasó los límites del derecho a la luz de las siguientes preguntas (Carmona, 2012): ¿puede un Estado mantener el orden y garantizar la seguridad a cualquier costo? ¿Para la administración política de la vida se puede utilizar cualquier herramienta, sin importar que con ello se la vulnere? ¿Puede esperarse de la administración política de la vida un atentado contra la vida misma con tal de que se cumpla su fin último - el mantenimiento del orden político y gubernamental一? ¿Hasta qué punto las acciones para negar y reducir la potencia de un sujeto hacen parte de los programas de gobiernos a fin de lograr aceptación, adhesión, administración y control de sus vidas?

Si la democracia como forma de gobierno se centra en la política y la estructura del Estado, este se asume como un organismo que se debe inmunizar de 
agentes externos. Así, todo lo que vaya contra el sistema se debe "extirpar" o impedir que se genere. Por esta vía, los falsos positivos son dispositivos inmunizadores que se emplean como condición necesaria para conservar la democracia. Díaz (2012) plantea seis dispositivos biopolíticos empleados en la implementación de la política de seguridad democrática: las interceptaciones telefónicas; la red de informantes; los soldados campesinos; el programa "vive Colombia, viaja por ella"; el pago a militares por rendimiento; $y$ los falsos positivos.

Pero, ¿qué es un falso positivo? En la estadística es un error mal llamado "de tipo alfa", al ser alfa la probabilidad de que ocurra este error. En la informática, es un error del sistema al encontrar un virus cuando en realidad no existe. En la medicina, es un error que enuncia una enfermedad cuando esta tampoco existe. Cualquiera de estas acepciones del término permite considerar, de manera general, que un falso positivo es suponer algo cuando en realidad no lo es. Para el caso colombiano, el término falso positivo hace referencia a un proceso por el cual el Ejército presentó a civiles desaparecidos como guerrilleros muertos en combate, tal como lo desarrollan Díaz, Salamanca y Carmona (2012).

El caso de los "falsos positivos" se enmarca y desarrolla durante el periodo de gobierno del presidente colombiano Alvaro Uribe Vélez (2002-2010), caracterizado por prácticas de muerte legitimadas en un discurso de seguridad para "vivir" en democracia. Así, se realizaron los asesinatos de jóvenes civiles por parte de integrantes de las Fuerzas Militares, y se presentaron ante la opinión pública como guerrilleros dados de baja en combate; más exactamente, como "positivos" en términos militares. La muerte de estos falsos combatientes conlleva una práctica de dominio de una forma de vida a través de la muerte, una "forma segura de vida" que ofrece el soberano a sus ciudadanos. Por esto deviene en una práctica biopolítica.

Así, entonces, son los falsos positivos en su práctica y denominación una muestra evidente de los dispositivos de control ejercidos desde un discurso biopolítico en el que el poder hegemónico busca: preservar la democracia como forma de gobierno; asegurar la seguridad de la democracia; acallar a través de la muerte; y promover de la democracia como estilo de vida. De allí que

Pensar que la democracia debe ser segura o que hay que asegurarla de cualquier manera o mejor, que hay que democratizar la seguridad son algunas de las múltiples interpretaciones que se desataron en la última década en Colombia. Esta fuerte y a veces peligrosa mezcla entre democracia y seguridad, llamó y enfiló muchas prácticas políticas del Estado y de la ciudadanía en Colombia. (Salamanca, 2012)

Una de estas prácticas son los asesinatos extrajudiciales o crímenes de lesa humanidad conocidos popularmente como "falsos positivos".

El recuento más cercano de este proceso de control social se ubica a finales del 2008, cuando empezaron a aparecer en varias regiones del país (Antioquia, Boyacá, Huila, Valle, Sucre y Norte de Santander) cadáveres de jóvenes que habían sido presentados por el Ejército Nacional como guerrilleros muertos en combate, como parte de la estrategia de lucha contra la guerrilla de las Fuerzas Armadas Revolucionarias de Colombia (FARC). El caso más emblemático corresponde a 19 jóvenes habitantes del municipio de Soacha, Cundinamarca, quienes habían desaparecido de sus lugares de origen. Todos fueron asesinados en condición de indefensión por parte de la Fuerza Pública. Estas ejecuciones extrajudiciales se conocieron en Colombia como "falsos positivos" del Ejército Nacional. Desde las investigaciones judiciales realizadas se han empezado a proferir sentencias condenatorias para algunos de los militares encontrados responsables de estos hechos.

Así, el asesinato de estos jóvenes se convirtió en un dispositivo de control y poder por parte del gobierno colombiano, por el cual se establece el pago de recompensas por captura o abatimiento en combate de guerrilleros o información para la captura de estos (Angarita, 2011); es decir, se ofreció un incentivo para acabar con la vida de quien era considerado enemigo y no adversario de una forma de gobierno. Política y judicialmente, estos casos se encuadran en los denominados "crímenes de Estado" o "terrorismo de Estado", entendidos como la "amenaza o uso de la violencia para lograr fines políticos, u otros por medio de la intimidación, la provocación, el miedo, y demás, dirigidos contra poblaciones civiles" (Chomsky, 2003).

De tal manera se puede reconocer que existió "una forma de poder que convirtió a la población y a la vida en el objeto primordial de su trabajo" (Sánchez, 2007).

Carmona (2012) plantea cómo, tal vez por eso, la mayoría de la población colombiana avaló la política de seguridad democrática que buscaba mantener el orden y la paz en el territorio colombiano al 
acabar con el enemigo, en este caso llamado "guerrillero", "terrorista" o "narcoterrorista", que en cualquiera de los casos era la excusa para legitimar el dispositivo causante de la muerte de decenas de seres humanos a quienes se disfrazaba de insurgentes. La legislación y la normatividad se adecuaron a dicha política: "El derecho se utilizó como instrumento por el soberano, para imponer su propia dominación” (Esposito: 2006). Más de 2000 bajas o "guerrilleros muertos en combate" fortalecieron el imaginario sobre la eficacia de una política de Estado fuerte y dura, y todo el sistema político se adecuó para la implementación del dispositivo de control y poder que permitió el ejercicio de la democracia como forma de gobierno. Ahora, un poco de tiempo después, la lectura la hacemos de otro modo.

\section{Conclusiones}

La psicología es política, dado el uso que se da a los conocimientos que genera. Por tanto, la psicología social también es política, sin que ello excluya la existencia de un área específica como es la psicología política.

Asumida de esta manera, la psicología social tiene como posibilidades de indagación e intervención categorías que "no le son propias", pero que en una migración interdisciplinar puede asumir, como son las categorías de "democracia" y "biopolítica".

La democracia, en un sentido general, se puede asumir en dos acepciones: como una forma de gobierno en la que se privilegia el abordaje de la política en su condición procedimental, y como estilo de vida. Es esta última se enfatiza en lo político y sus desdoblamientos procesuales.

La fuerza de la propuesta que ayudamos a desarrollar en el presente texto tiene que ver con asumir la democracia como una construcción colectiva, histórica y procesual que se despliega en la vida cotidiana tanto en los ámbitos públicos como privados.

En cuanto colectiva, la democracia no es una construcción individual del soberano que excluye voces polifónicas y asume lo diferente como enemigo, sino que se puede instituir como estilo de vida desde el cual se reconoce y potencia la otredad en cuanto antagónico, y por vía del pólemos se ayuda en la constitución de una política agonística. Ayudar en el fortalecimiento de la democracia como forma de gobierno implica hacer otro tanto con la democracia como estilo de vida, para lo cual se requiere pensar en procesos de educación para la democracia que ayuden en la constitución de sujeto político.

Los casos de los crímenes de lesa humanidad son una muestra de las maneras como el soberano, al asumir la democracia solo como forma de gobierno, reconoce en el contradictor un enemigo al que se debe eliminar físicamente. Para esto se acude a la inmunización del sistema mediante la generación del temor que emerge de la muerte concretada mediante un dispositivo de control y poder, como lo son los falsos positivos.

\section{Referencias}

Arendt, H. (1954). La condición humana. Buenos Aires: Paidós.

Andrade-Salazar, J. A. (2013). La intervención clínico social en la sociedad biopolítica. Revista de Psicología GEPU, 4(1), 166-179. Recuperado de https://goo.gl/7AAFyp

Angarita, P. (2011). Seguridad democrática. Lo invisible de un régimen politico y económico. Bogotá: Siglo del Hombre y Universidad de Antioquia.

Allport, G. (1937). Personality. A psychological interpretation. Nueva York: Henry Holt.

Baró, I. (1988). Acción e ideología. Psicología social desde Centroamérica. San Salvador: UCA.

Bartolomé, C. (2012). El homo sacer y la ciudad antigua. Abandono y gobierno de la vida humana, un diálogo con Giorgio Agamben. En C. Bartolomé y R. Duarte (Coor.), La urbe global y el gobierno de la vida humana. Justicia, alteridad y memoria en los espacios de poder (pp. 25-48). Bogotá: Universidad Libre, Asociación Iberoamericana de Filosofía Política.

Brandao, M. (2010). Democracia e psicologia social crítica. Psico, 41(3), 317-324. Recuperado de https://goo.gl/ cRSzTv

Bruno, D. y Barreriro, A. (2015). La representación social de la democracia de adolescentes argentinos. Escritos de Psicología, 8(3), 33-40. doi: 10.5231/psy. writ.2015.1506.

Carmona, O. (2012). Los falsos positivos como crímenes de Estado: un análisis desde la biopolítica y el bioderecho. En C. Bartolomé y R. Duarte (Coords.), La urbe global y el gobierno de la vida humana. Justicia, alteridad y memoria en los espacios de poder (pp. 195-206). Bogotá: Universidad Libre, Asociación Iberoamericana de Filosofía Política.

Castillo, A. y Osorio, J. (1997). Dimensiones educativas de la construcción de ciudadanía. Foro, 32, 90-98. 
Chomsky, N. (2003). Poder y terror. Barcelona: RBA.

Cruz, M. (2017). De la biopolítica a la psicopolítica en el pensamiento social de Byung-Chul Han. Athenea Digital, 17(1), 187-203. doi: https://doi.org/10.5565/ rev/athenea. 1782

Dewey, J. (1995). Democracia y educación. Madrid: Morata.

Díaz, Á, y Valencia, G. (1996a). Educación y democracia. En Silvio Cardona (Comp.). Educación y sociedad. Lecturas desde la Universidad Católica de Manizales (pp. 68-153). Manizales: Editorial Universidad Católica de Manizales.

Díaz, Á, y Valencia, G. (1996b). Potencial político de la democracia como estilo de vida. En: Temas fundamentales para la educación en el siglo XXI (pp. 55-64). Manizales: Editorial Universidad Católica de Manizales.

Díaz, Á. (1999). La democracia como estilo de vida o una nueva perspectiva para pensar y actuar hacia una educación para la democracia. En Á. Díaz, Bitácora. Ensayos sobre educación para la ciudadanía (pp. 48-57). Manizales: Editorial Universidad Católica de Manizales.

Díaz, Á. (2000a). Aportes pedagógicos en educación y democracia. Hojas Universitarias, 49, 40-48.

Díaz, Á. (2000b). Conceptos y prácticas sobre la democracia en un grupo de maestras y maestros de básica primaria, de la ciudad de Manizales. En. Á. Díaz (Comp.), Espacios democráticos en el ámbito escolar (pp. 8-26). Manizales: Editorial Universidad Católica de Manizales.

Díaz, Á. (2003). Una discreta diferenciación entre la política y lo político y su incidencia sobre la educación en cuanto socialización política. Reflexión política, 9(5), 49-61. Recuperado de https://goo.gl/c5XEdt

Díaz, Á. (2012). Hacer morir y dejar vivir... hacer vivir y dejar morir: El caso de los "falsos positivos". En C. Bartolomé y R. Duarte (Coords.), La urbe global y el gobierno de la vida humana. Justicia, alteridad y memoria en los espacios de poder (pp. 171-180). Bogotá: Universidad Libre, Asociación Iberoamericana de Filosofía Política.

Díaz, A., Salamanca, L., y Carmona, O. (2012). Biopolítica, subjetividad política y falsos positivos. En C. Piedrahita, A. Díaz y P. Vommaro (Comp.), Subjetividades políticas: desafíos y debates latinoamericanos (pp. 47-62). Bogotá: Universidad Distrital Francisco José de Caldas, Clacso.

Esposito, R. (2006). Bíos. Biopolítica y filosofía, Buenos Aires: Amorrortu.
Foucault, M. (2008). Nacimiento de la biopolítica. Buenos Aires: Fondo de Cultura Económica.

Foucault, M. (1984). Historia de la sexualidad I. El uso de los placeres. Buenos Aires: Fondo de Cultura Económica.

Grajales, A. (2014). Los falsos positivos como dispositivos biopolíticos de guerra psicológica. Recuperado de http://repositorio.ucp.edu.co:8080/jspui/ bitstream/10785/3015/1/CDMPSI213.pdf

Hederich, C. (2007). Estilos cognitivos en la dimensión de dependencia. Independencia de campo. Influencias culturales e implicaciones para la educación. Bogotá: Universidad Pedagógica Nacional.

Mendiola, I. (2009). La biopolítica como un pensar fronterizo. España: Anthropos.

Mélich, J. (1994). Del extraño al cómplice. La educación en la vida cotidiana. Barcelona: Anthropos.

Mouffe, Ch. (1999). El retorno de lo político. Barcelona: Paidós.

Mouffe, Ch. (2000). La paradoja democrática. Barcelona: Gedisa.

Montero, M. (2004). Introducción a la psicología comunitaria. Buenos Aires: Paidós.

Mora, D. (2012) Formación democrática y escuelas democráticas para la construcción de ciudadanía crítica. Recuperado de http://www.scielo.org.bo/scielo.php? script=sci_arttext\&pid=S1997-40432012000200002

Pastor, J. (2009). Relevancia de Foucault para la psicología. Psicothema, 21(4), 628-632. Recuperado de https:// goo.gl/hyccBd

Porras, N. (2010). Psicología y educación para la democracia: una reflexión para la formación ciudadana. Recuperado de http://www.funlam.edu.co/ revistas/index. php/poiesis/article/view/56/28

Salamanca, L. (2012). El biopoder y los falsos positivos como dispositivo de control y vigilancia. En C. Bartolomé y R. Duarte (Coords.), La urbe global y el gobierno de la vida humana. Justicia, alteridad y memoria en los espacios de poder (pp. 181-193). Bogotá: Universidad Libre, Asociación Iberoamericana de Filosofía Política.

Sánchez, R. (2007). Biopolítica y formas de vida. Bogotá: Pontificia Universidad Javeriana.

Seoane, J., Mougán, J., y Lago, J. (2009). La democracia como un estilo de vida. Madrid: Siglo XxI.

Toscano, D. (2008). Recensión del libro. Nacimiento de la biopolítica. Papel Político, 13(2), 730-735

Ugarte, J. (Comp.). (2005). La administración de la vida. Estudios biopolíticos. Barcelona: Anthropos. 